\title{
INVERSIÓN PÚBLICA Y POBREZA MONETARIA EN LAS FAMILIAS DE LA REGION DE PUNO - PERÚ (2004 - 2019)
}

\author{
PUBLIC INVESTMENT ON MONETARY POVERTY IN FAMILIES IN THE PUNO \\ REGION - PERU, $2004-2019$
}

Julio Cesar QUISPE MAMANI $^{1}$

Felix QUISPE MAMANI ${ }^{2}$

Marcial GUEVARA MAMANI ${ }^{3}$

Ronald Raúl ARCE COAQUIRA ${ }^{4}$

Cristóbal Rufino YAPUCHURA SAICO ${ }^{5}$

Alberto CATACHURA VILCA ${ }^{6}$

\begin{tabular}{|lll|}
\hline Recibido & $:$ & 27.10 .2020 \\
Aceptado & $:$ & 28.12 .2020 \\
Publicado & $:$ & 12.02 .2021 \\
\hline
\end{tabular}

RESUMEN: El objetivo fue analizar el efecto de la inversión pública en la pobreza monetaria de las familias de la Región de Puno, por lo cual se consideró la metodología descriptiva y correlacional, aplicando el modelo Log-lineal y Log-Log. Se determinó que la inversión pública realizada por el Estado en Puno, entre el periodo de 2004-2019, en promedio tuvo una tasa de crecimiento de $23.58 \%$ anualmente y el nivel de pobreza disminuyo en promedio $5.16 \%$ por año. Se concluye estableciendo que la inversión pública si tuvo un efecto directo en la disminución de la pobreza monetaria en las familias de la Región de Puno; el efecto que tuvo la inversión pública en el sector de saneamiento y vivienda, educación y salud en la pobreza monetaria es positivo, ya que a un incremento de $1 \%$ en la inversión pública en el sector de saneamiento y vivienda, la probabilidad de que la pobreza monetaria disminuya es de $2.59 \%$ y un incremento de $1 \%$ en la inversión pública en el sector educación, entonces la probabilidad de que la pobreza monetaria disminuya es de $8.75 \%$.

Palabras clave: Inversión pública, educación, salud, viviendas y pobreza monetaria.

ABSTRACT: The objective was to analyze the effect of public investment on the monetary poverty of families in the Puno Region, for which the descriptive and correlational methodology was considered, applying the Log-linear and Log-Log models. It was determined that the public investment made by the State in Puno between the periods from 2004 to 2019 on average had a growth rate of $23.58 \%$ annually and the poverty level decreased on average $5.16 \%$ per year. It is concluded by establishing that public investment did have a direct effect on the reduction of monetary poverty in families in the Puno Region; The effect that public investment in the sanitation and housing, education and health sector had on monetary poverty is positive, since at an increase of $1 \%$ in public investment in the sanitation and housing sector, the probability that the Monetary poverty will decrease is $2.59 \%$ and an increase of $1 \%$ in public investment in the education sector, then the probability that monetary poverty will decrease is $8.75 \%$.

Keywords: Public investment, education, health, housing and monetary poverty.

\footnotetext{
${ }^{1}$ Magíster en Desarrollo Regional y Medio Ambiente. Universidad Nacional del Altiplano. Puno-Perú. Email: jcquispe@unap.edu.pe. ORCID: https://orcid.org/0000-0002-3938-1459

${ }^{2}$ Magister en Ciencias Sociales. Universidad Nacional del Altiplano. Puno-Perú. Email: felix.x.21@ hotmail.com. ORCID: https://orcid.org/0000-0003-1578-8315

${ }^{3}$ Magíster en Economía. Universidad Nacional del Altiplano. Puno-Perú. Email: guevaraunap@ gmail.com. ORCID: https://orcid.org/0000-0003-3545-1306

${ }_{4}^{4}$ Magíster en Economía. Universidad Nacional del Altiplano. Puno-Perú. Email: arceroni1@ hotmail.com. ORCID: https://orcid.org/0000-0002-6546-0038

${ }^{5}$ Doctor en Administración. Universidad Nacional del Altiplano. Puno-Perú. Email: cyapuchura@unap.edu.pe. ORCID: https://orcid.org/0000-0003-1956-3922

${ }^{6}$ Magíster en Gestion y Desarrollo del Turismo Rural. Universidad Nacional del Altiplano. Puno-Perú. Email: albertocatachura@unap.edu.pe ORCID: https://orcid.org/0000-0002-3033-3696
} 


\section{Journal of the Academy $|125|$}

\section{INTRODUCCIÓN}

En las últimas décadas el nivel de pobreza ha ido mostrando una brecha amplia y divergente, la misma que es impulsada por los inconvenientes que tienen las familias en los aspectos socioeconómicos y el rol del Estado cobra una preponderancia hacia la lucha de la misma, a fin de combatir hacia su disminución mediante la implementación de las políticas sociales, económicas y publicas principalmente (Anderson et al., 2006; De Anda, 2013; Vásquez, 2012). El problema de la pobreza es muy complejo y a la vez traspasa transversalmente todos los estatus sociales que pueda existir en una sociedad, estando asociada por las diferentes variables que explican su comportamiento y a través de las mismas se puede proponer algunas políticas para disminuir o contribuir hacia la disminución de esta (Herrera y Reyes, 2020; Ortega, 2016; PNUD, 2000; Ramió y Salvador, 2005).

Dentro de las funciones establecidas por el Estado es brindar las condiciones y los servicios necesarios para garantizar una calidad de vida adecuada de las personas que forman parte de la nación, y esto se alcanza mediante el diseño, la formulación e implementación de adecuadas políticas económicas y sociales (Gutiérrez, 2015; D’Amico, 2015; Figueroa, 2014; Salazar y Chacaltana, 2018).

Como resaltan Arias y Sucari (2019), la educación tiene un efecto positivo sobre la disminución del nivel de pobreza monetaria, debido a que la educación primaria que agrupa a una parte de la Población Económicamente Activa (PEA) conlleva a la reducción de la pobreza al 0.99\%, la PEA con educación secundaria contribuye a la disminución de la pobreza en $1.57 \%$ y la educación superior no universitaria contribuye a la reducción de la pobreza en $0.91 \%$ respectivamente; de ahí resalta la importancia de los instrumentos de gestión pública para alcanzar la eficiencia tanto en el uso y distribución de los recursos del Estado mediante la realización de una inversión pública integral en los diferentes sectores de servicios públicos y productivos ya sea de educación, salud, vivienda, transportes, agricultura, etc.

De acuerdo a Tuiz y Duarte (2015), la inversión pública busca alcanzar la eficiencia en la ejecución presupuestal de los proyectos y programas, cumpliendo las metas programadas en el tiempo previsto. Establecer una interrelación vertical entre planes, programas y proyectos, dentro de un proceso administrativo público y privado (Gómez y Tacuba, 2017). En este sentido, esta 


\section{Journal of the Academy $|126|$}

participación del Estado en la sociedad es más compleja en los países subdesarrollados como el Perú, donde en las últimas décadas ha experimentado un importante crecimiento económico, contribuyendo hacia la generación de mayores recursos fiscales, los cuales han permitido la realización de mayores inversiones públicas a fin de garantizar con mejores condiciones de infraestructura y servicios públicos, sin embargo no fue suficiente para generar mejores impactos, en vista que se muestra una desigualdad en la distribución de los recursos (Aramburú y Rodríguez, 2011; Barragán y Roemer, 1999; CEPAL, 2020; Cruces y Gasparini, 2013; Romero, 2000).

En 2018, el 20.5\% de la población del Perú fueron situados en situación de pobreza, demostrando de esta manera que disminuyo solo en 1,2\% con respecto al año 2017 (INEI, 2020; Sánchez, 2015); evidencia que la participación del Estado mediante la dinamización de la inversión pública que lo realiza a través de los 1874 municipios (1678 distritales y 196 provinciales) no fueron efectivas ni eficientes, en vista que no cumplieron con las tareas de influenciar hacia la disminución de la pobreza monetaria en las familias mediante el gasto público eficiente (Jiménez y Sosa, 2018).

El nivel de pobreza a nivel de regiones es más desigual, debido a los diferentes aspectos que en estas repercuten; en la Región de Puno el nivel de pobreza asciende en promedio al 37\%, incorporando una pobreza extraña del $6.3 \%$ y casi el $41 \%$ de la población total forman parte del grupo de vulnerables, toda vez que solo el $43 \%$ del total de población tiene acceso a los servicios de agua y saneamiento, salud, educación y energía eléctrica (Arpi, 2017; INEI, 2020; Paredes y Escobar-Mamani, 2018). Complementariamente la ejecución de los gastos que considera desde el compromiso, devengado y girado en el sector social al 2019 fue deficiente, donde solo el 12\% del presupuesto asignado en las actividades correspondientes al desarrollo infantil temprano fueron ejecutados, solo $13 \%$ del presupuesto asignado al desarrollo integral de la niñez y la adolescencia, el 6\% del presupuesto asignado a las actividades de inclusión económica y solo $11 \%$ del presupuesto asignado a la protección del adulto mayor (Ccama, 2018; Romero, 2000; Tumi y Escobar, 2018).

Estos indicadores muestran la realidad critica en la que se encuentran la Región de Puno, toda vez que la baja ejecución en las acciones y programas como parte de la inversión pública no aportan de manera contundente hacia la mejora de la calidad de vida de la población puneña, 


\section{Journal of the Academy $|127|$}

disminuyendo en el nivel de pobreza; del cual, las familias buscan sobresalir de alguna manera, implementando actividades de subsistencia (productivas o de servicios), que les permitan contrarrestar de alguna manera esta situación (Fort y Paredes, 2015; Ocas, 2019). El nivel de calidad de vida de las familias tiene mucho que ver con el ingreso económico que estos generan, toda vez que refleja en el comportamiento de las variables económicas y sociales asociadas con la pobreza (Rodríguez y Sanchez, 2017). Así como indican Urbina-Padilla y Quispe (2016), acceder a contar con los servicios necesarios de la vivienda es importante para el desarrollo social familiar y por ende es una determinante del nivel de pobreza, toda vez que al proporcionar y cubrir las necesidades básicas de la familia mediante el servicio de agua potable y saneamiento, servicio de energía eléctrica y telefonía de comunicación, entonces la probabilidad de disminuir el nivel de pobreza es alto.

Por lo tanto, se muestra que la pobreza es uno de los graves problemas que aqueja a la Región de Puno desde tiempos remotos hasta la actualidad, convirtiéndose en un mal que sin duda ha calado con mayor fuerza en los últimos años, donde a pesar que algunas familias cuentan con los servicios en salud, educación, vivienda y saneamiento básico, aún existen familias en pobreza y pobreza extrema (Tinuco et al., 2018). El crecimiento sostenido no es suficiente para erradicar la pobreza, debido a que no se refleja en una inversión pública eficiente y que permita cerrar las brechas sociales y disminuir la pobreza monetaria de las familias; lo que llevó a plantear la siguiente interrogante general: ¿Cuál es el efecto de la inversión pública en la pobreza monetaria en las familias de la Región de Puno?, además de las preguntas específicas de ¿Qué efecto tiene la inversión del sector de saneamiento y vivienda, educación y salud en la pobreza monetaria en las familias de la Región de Puno?. Complementariamente la investigación busca comprobar la hipótesis general: La inversión pública tiene un efecto directo en la disminución de la pobreza monetaria en las familias y la hipótesis específica: El efecto que tiene la inversión del sector de saneamiento y vivienda, educación y salud en la pobreza monetaria en las familias de la región de Puno es positivo.

\section{DESARROLLO}

\section{Materiales y métodos}

La presente investigación corresponde al tipo de investigación descriptivo y correlacional, cuya unidad de análisis es determinar la relación existente entre la inversión pública con la pobreza 
monetaria en la Región de Puno, cuyo periodo de estudio son entre 2004 al 2019. Corresponde al diseño no experimental y de series de tiempo (Hernández et al., 2017).

\section{Población y Muestra}

\section{Población}

Se tomó como población objeto de estudio a los que radican en la Región de Puno, que asciende a 1,172,697 habitantes, de la cual, se considera en el reporte histórico de datos pobreza monetaria e inversión pública (Solano y Álvarez, 2005).

\section{Muestra}

Considerando que el análisis de la investigación es con información histórica desde el 2004 al 2019, entonces la muestra corresponde a los datos históricos existentes en pobreza monetaria, inversión pública total, inversión en el sector educación, inversión en el sector salud y la inversión en el sector saneamiento y vivienda en dicho periodo, cuya fuente de información es el Ministerio de Economía y Finanzas (MEF) e Instituto Nacional de Estadística e Informática (INEI) (Lacort, 2014).

\section{Identificación De Variables}

La presente investigación, toma como variables la pobreza monetaria expresada porcentualmente y la inversión pública expresada en soles. Se consideró a la inversión pública total, la ejecutada en los diferentes sectores que este atañe; además de la inversión realizada en los proyectos específicos del sector de saneamiento y vivienda, educación y salud (Hernández-Sampieri y Torres, 2018).

\section{Fuentes De Información}

La presente investigación toma como principal fuente secundaria, donde a través de la recopilación de información estadística proporcionada por el Ministerio de Economía y Finanzas del Perú (MEF), el Instituto Nacional de Estadística e Informática (INEI), y la Encuesta Nacional de Hogares (ENAHO), que consideran los registros históricos de las transferencias económicas realizadas al Gobierno Regional de Puno; así como la sistematización de información mediante revistas, informes, artículos, tesis, folletos y demás publicaciones referidas a la presente investigación (Solano y Álvarez, 2005). 


\section{Journal of the Academy $|129|$}

\section{Análisis de variables}

Variable endógena

PMt

Variables exógenas

(log)InvpTt, (log)Invedut, (log)Invsat, (log)Invivit

Donde:

$P M t$ es la pobreza monetaria representado en porcentualmente.

InvpTt es la inversión pública total en soles.

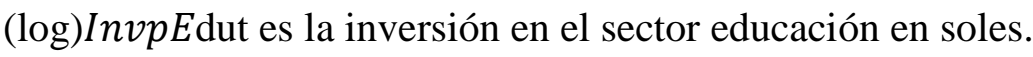

( $\log$ InvpSat es la inversión en el sector salud en soles.

$(\log )$ Invivit es la inversión en el sector saneamiento y vivienda en soles.

El monto de las inversiones consideradas en forma general y por sectores evidencia a nivel de devengado en el Sistema de Administración Financiera (SIAF) del MEF.

\section{Planteamiento del modelo}

Para establecer el efecto de la inversión pública en la disminución de la pobreza monetaria en las familias se consideró un modelo econométrico de regresión log-lineal, donde las variables consideradas fueron la $P M \mathrm{t}$ que es la pobreza monetaria representado en porcentualmente y la InvpTt que es la inversión pública total en soles.

En este sentido, la representación econométrica es igual a:

$$
\mathrm{PMt}=\alpha-\beta \operatorname{InvpTt}+\mathrm{e}
$$

Para el caso de determinación del efecto que tiene la inversión del sector de saneamiento y vivienda, educación y salud en la pobreza monetaria en las familias de la región de Puno se consideró el siguiente modelo econométrico de tipo regresión lineal Log-Log, la misma que es atribuible a las elasticidades que se considera de la inversión total, en el sector educación, salud y saneamiento y vivienda en la pobreza monetaria.

$$
\text { PMt }=\propto-\beta(\log ) \operatorname{InvpEdu}-\delta(\log ) \operatorname{InvpSat}-\theta(\log ) \text { Invivit }+\mathrm{e}
$$




\section{Journal of the Academy $|130|$}

\section{Procesamiento y tabulación de datos}

Para el procesamiento, tabulación de los datos y la prueba de las hipótesis, se utilizó los paquetes econométricos como el SPSS 25.0 y EVIEWS 10.0.

\section{Resultados y discusión}

Considerando la teoría económica, la relación existente entre la inversión pública y la pobreza monetaria es inversa, por lo cual, de acuerdo a los antecedentes revisados, es decir frente a un aumento de inversión pública se espera que la pobreza monetaria disminuya. En el periodo de análisis, el comportamiento de la inversión pública en la Región de Puno fue muy variante, en vista que desde el 2007 en adelante incremento fuertemente la asignación presupuestal, mostrando una inversión al 2008 de 600,998,361 soles, con un incremento de $88.20 \%$ con respecto al año anterior, y esto se ha ido manteniendo para los próximos años. Esta variación al 2016 llego a alcanzar a 1,593,571,356 soles de inversión, representando un incremento de $22.99 \%$ respecto al 2015 y a pesar de mostrar una permanencia en el monto invertido entre los años 2018 y 2019 de $3.02 \%$ y de $-8.20 \%$ de disminución con respecto al año anterior, esta sigue siendo alto a comparación del 2004; y en promedio en el periodo de análisis se tuvo una tasa de crecimiento de $23.58 \%$ (MEF, 2020) (Figura 1). 


\section{Journal of the Academy $|131|$}

\section{Figura 1.}

Comportamiento de la inversión pública en la Región de Puno

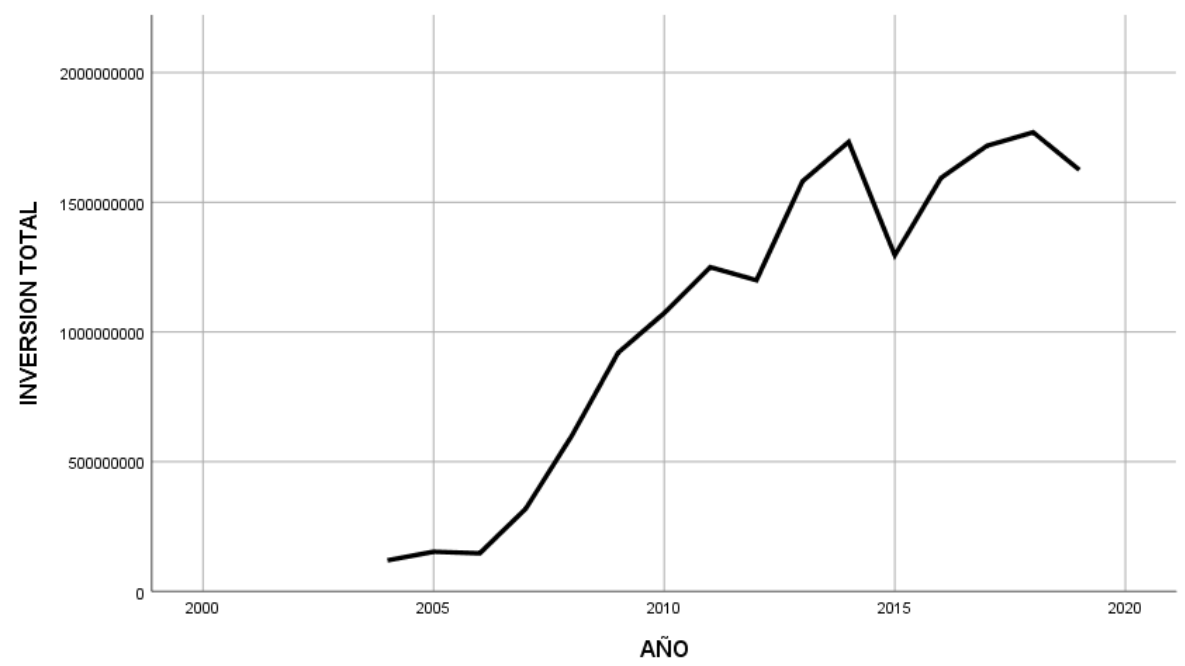

Nota: Elaboración propia en base a información del MEF (2004-2020)

En este sentido, a pesar de realizar inversiones en proyectos y programas de inversión considerables, estas no han aportado de manera contundente en los indicadores de pobreza, en vista que la calidad de vida de la mayor parte de la población que radica en la región no muestran cambios significativos hasta la actualidad, tal es el caso que en el periodo de análisis en el 2004, Puno tenía un nivel de pobreza que estaba en $79.3 \%$, en el 2010 esta disminuyó a 48.6\%, llegando al 2019 a 34.4\%, del cual en promedio disminuyó por año en 5.16\%. Las tasas de disminución del nivel de pobreza en los últimos años fueron muy ínfimas, toda vez que su contribución a esta no se mostró con el mismo impacto en los años anteriores, demostrando que no se ha planteado de manera óptima la asignación de los recursos en acciones de vital importancia como es en los sectores de vivienda y saneamiento, en educación y salud (MEF, 2020) (Figura 2). 


\section{Figura 2.}

Tasa de disminución del nivel de pobreza en la Región de Puno

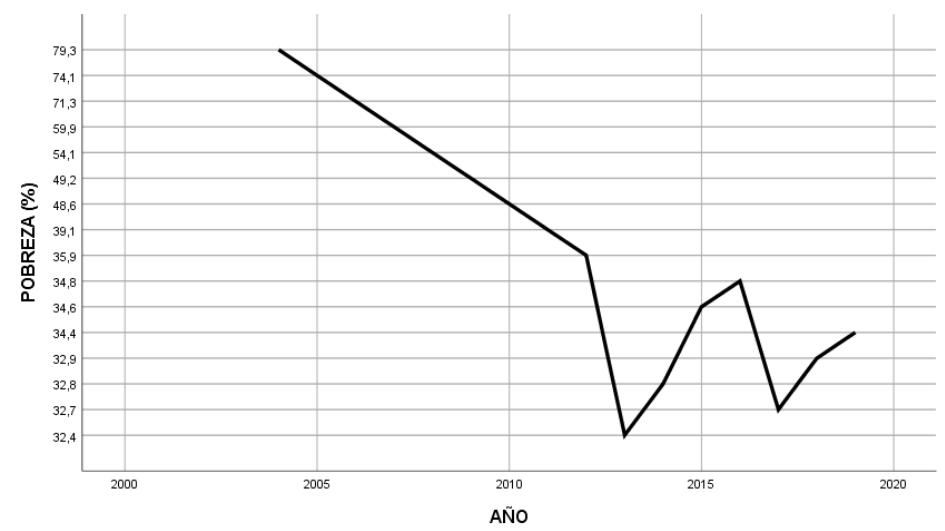

Nota: Elaboración propia en base a información del MEF (2004-2020)

Por lo cual, considerando el sustento vertido en los puntos anteriores, se puede determinar que las variaciones de la pobreza monetaria si están explicados por la inversión pública que realiza en Estado en la Región de Puno, toda vez que mayores inversiones mostraron como efecto una disminución en el nivel de pobreza.

Realizando la prueba de hipótesis de la investigación, donde en la primera se establece que como Ho: La inversión pública no tiene un efecto directo en la disminución de la pobreza monetaria en las familias de Puno, en el periodo 2004-2019 y la Ha: La inversión pública tiene un efecto directo en la disminución de la pobreza monetaria en las familias de Puno, en el periodo 20042019. Se aplicó un modelo de regresión Log - lineal, donde la variable independiente que es la inversión pública y la dependiente es la pobreza monetaria.

Al realizar la regresión se obtuvo los resultados considerados en la tabla 1, donde la inversión pública explica en $92.77 \%$ a la pobreza monetaria en las familias de Puno y al contrastar el Fcalculado que asciende a 179.86 con el F-Tabla: 3.44, se evidencia que el F-calculado > F-Tabla, del cual a un nivel de confianza del $95 \%$ o $5 \%$ de significancia se acepta la Ha (Figura 3), concluyendo que la inversión pública si tuvo un efecto directo en la disminución de la pobreza monetaria en las familias de Puno en el periodo 2004-2019, toda vez que a un incremento de 1 
sol en la inversión pública, entonces la probabilidad de que la pobreza monetaria disminuya es de $2.57 \mathrm{E}-08$.

\section{Figura 3.}

Prueba F de Fisher para modelo 1

$$
\begin{aligned}
& K=4 \\
& n=16
\end{aligned}
$$

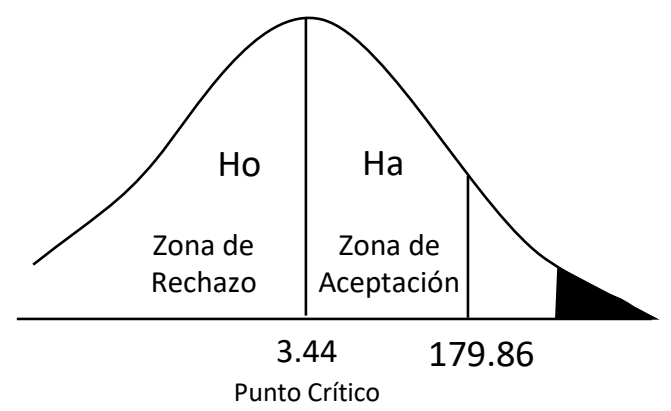

Nota: Elaboración propia

\section{Tabla 1.}

Regresión de modelo econométrico 1

\begin{tabular}{lrlrr}
\hline \multicolumn{1}{c}{ Variable } & \multicolumn{2}{c}{ Coefficient Std. Error } & t-Statistic & Prob. \\
\hline \multicolumn{1}{c}{ INVPT } & -2.57 E-08 & 1.92 E-09 & -13.41124 & 0.0000 \\
\multicolumn{1}{c}{ C } & 74.09715 & 2.345554 & 31.59047 & 0.0000 \\
\hline R-squared & 0.927783 & Mean dependent var & 46.63125 \\
Adjusted R-squared & 0.922625 & S.D. dependent var & 16.44245 \\
S.E. of regression & 4.573691 & Akaike info criterion & 5.994987 \\
Sum squared resid & 292.8610 & Schwarz criterion & 6.091560 \\
Log likelihood & -45.95989 & Hannan-Quinn criter. & 5.999932 \\
F-statistic & 179.8612 & Durbin-Watson stat & 1.236912 \\
Prob(F-statistic) & 0.000000 & & \\
\hline
\end{tabular}

Nota: Elaboración propia en base a información del MEF (2004-2020)

La inversión pública por sectores requeridos y su adecuada orientación para la toma de decisiones es importante, ya que este actuar permitirá la obtención de una mejor asignación de los recursos 
del Estado en la sociedad. En la Región de Puno se hizo inversiones considerables en el sector de saneamiento y vivienda, educación y salud, pero no con proyectos o programas de envergadura mayor o de impacto regional, complementado con el descuido en el sector productivo, quizá esa sea el motivo por la cual no se contribuyó de manera categórica en la disminución del nivel de pobreza.

Para establecer el efecto que tiene la inversión del sector de saneamiento y vivienda, educación y salud en la pobreza monetaria en las familias de la Región de Puno se aplicó el modelo de regresión $\log$ - Log, donde se muestra que las variables más significativas son la inversión pública en el sector saneamiento y vivienda y la inversión en la educación, mas no en el sector de salud. Además, considerando un nivel de confianza de 95\%, las variables inversión de educación y la inversión en el sector saneamiento y vivienda explican a la pobreza monetaria en las familias de Puno en un $98.67 \%$ (Tabla 2).

\section{Tabla 2.}

Regresión de modelo econométrico 2

\begin{tabular}{llccl} 
Variable & Coefficient & Std. Error & t-Statistic & Prob. \\
\hline LOG(INVSA) & 0.535938 & 0.920178 & 0.582428 & 0.5746 \\
LOG(INVIVI) & -2.592546 & 0.580431 & -4.466590 & 0.0016 \\
LOG(INVEDU) & -8.753384 & 2.040151 & -4.290558 & 0.0020 \\
C & 242.7062 & 34.71354 & 6.991688 & 0.0001 \\
AR(1) & 0.437850 & 0.525927 & 0.832529 & 0.4266 \\
MA(1) & 0.423269 & 0.662565 & 0.638834 & 0.5388 \\
SIGMASQ & 3.358131 & 1.860470 & 1.804991 & 0.1046 \\
\hline R-squared & 0.986751 & Mean dependent var & 46.63125 \\
Adjusted R-squared & 0.977918 & S.D. dependent var & 16.44245 \\
S.E. of regression & 2.443361 & Akaike info criterion & 4.971155 \\
Sum squared resid & 53.73010 & Schwarz criterion & 5.309163 \\
Log likelihood & -32.76924 & Hannan-Quinn criter. & 4.988464 \\
F-statistic & 111.7135 & Durbin-Watson stat & 1.757975 \\
Prob(F-statistic) & 0.000000 & &
\end{tabular}

Nota: Elaboración propia en base a información del MEF (2004-2020)

Al realizar la prueba de hipótesis, donde se consideró como Ho es que el efecto que tiene la inversión en el sector de saneamiento y vivienda, educación y salud en la pobreza monetaria en las familias de la región de Puno es negativo, mientras que la Ha es que el efecto que tiene la 
inversión en el sector de saneamiento y vivienda, educación y salud en la pobreza monetaria en las familias de la región de Puno es positivo.

Contrastando el F-calculado que asciende a 111.71 con el F-Tabla que es igual a 4.60 se evidencia que el F-calculado > F-Tabla, determinando que se rechaza la Ho y se acepta la Ha (Figura 4), concluyendo que el efecto que tuvo la inversión pública en el sector de saneamiento y vivienda, educación y salud en la pobreza monetaria en las familias de la región de Puno es positivo (Tabla 2). Del cual, a un incremento de $1 \%$ en la inversión pública en el sector de saneamiento y vivienda, entonces la probabilidad de que la pobreza monetaria disminuya es de 2.59\%; un incremento de $1 \%$ en la inversión pública en el sector educación, entonces la probabilidad de que la pobreza monetaria disminuya es de $8.75 \%$ (Tabla 2).

Figura 4. Prueba F de Fisher para modelo 2

$\mathrm{K}=2$
$\mathrm{n}=16$

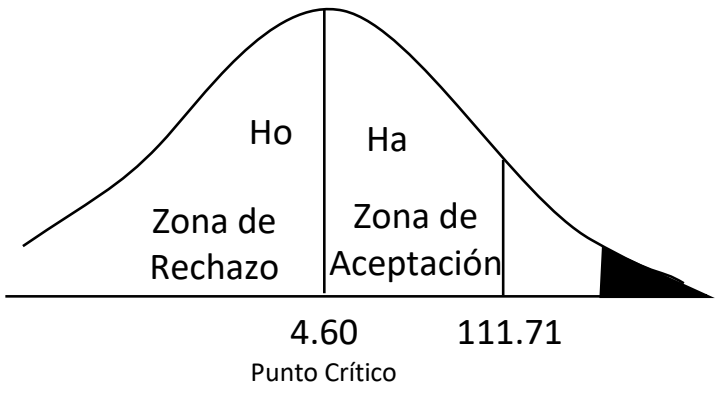

Nota: Elaboración propia

En este sentido, los resultados obtenidos en la presente investigación son coincidentes con Alvarado et al. (2019) donde al aplicar un modelo de Mínimos Cuadros Ordinarios para determinar el impacto de la inversión social en la pobreza pudo establecer que esta es explicada hacia una disminución de la pobreza en $21.34 \%$ en la zona rural y urbano en Ecuador, con mayor impacto en la zona rural. Es coincidente también con el análisis realizado por Fort y Paredes (2015), donde determinan que la implementación de la inversión pública en sistemas riego, caminos vecinales, comunicación y proyectos productivos tienen mayor significancia sobre la reducción de la pobreza en las zonas rurales.

La inversión pública que realiza en Estado a través de los proyectos y programas de inversiones en los servicios básicos de las viviendas contribuyen de manera categórica en la disminución de 


\section{Journal of the Academy $|136|$}

la pobreza monetaria y esto sostenido por las acciones en el sector salud que coincide con Urbina y Quispe (2016). Quizá comparando con Orco (2009), los resultados que se obtuvo en la presente investigación no son tan absolutos, en vista que dicho autor al analizar el impacto del gasto público y las inversiones en la reducción de la pobreza, estas contribuyen en $43.23 \%$ y es mucho mayor por la amplitud de estudio y el ámbito de intervención que es a nivel del Perú pero tienen la misma orientación.

Finalmente, la investigación coincide con lo investigado por Hallasi (2019), al concluir también que una mayor inversión en el sector social, permitirá en disminuir la pobreza en 0.09\%; al igual que con Aguedo y Romero (2018) donde pudieron determinar el impacto de la inversión pública sobre la pobreza en 5.75\%. Según Ylla (2019) la inversión pública pudo contribuir en la pobreza en $70.5 \%$ y en la pobreza extrema en $58.9 \%$, concordante con Vélez (2018); donde pudo determinar el impacto de la inversión pública sobre la pobreza medido a través del coeficiente de Gini entre los periodos de 2014 - 2017 que fue de $0.49 \%$ en el 2014 y $0.46 \%$ en el 2017, disminuyendo en $3 \%$.

\section{CONCLUSIONES}

La inversión pública realizada por el Estado en la Región de Puno entre el periodo de 2004 al 2019 en promedio tuvo una tasa de crecimiento de $23.58 \%$ anualmente, por el contrario, el nivel de pobreza en el 2004 fue de 79.3\%, llegando a disminuir al 2019 a 34.4\%, del cual en promedio disminuyo por año en $5.16 \%$, mostrando una disminución en los últimos años a una tasa mínima sin impacto sobre la población. Por lo que se determina que la inversión pública sí tuvo un efecto directo en la disminución de la pobreza monetaria en las familias de la Región de Puno en el periodo 2004-2019, toda vez que, a un incremento de 1 sol en la inversión pública, entonces la probabilidad de que la pobreza monetaria disminuya es de 2.57E-08.

Además, el efecto que tuvo la inversión pública en el sector de saneamiento y vivienda, educación y salud en la pobreza monetaria en las familias de la región de Puno es positivo, toda vez que a un incremento de $1 \%$ en la inversión pública en el sector de saneamiento y vivienda, entonces la probabilidad de que la pobreza monetaria disminuya es de $2.59 \%$; un incremento de $1 \%$ en la inversión pública en el sector educación, entonces la probabilidad de que la pobreza monetaria disminuya es de $8.75 \%$. 


\section{REFERENCIAS BIBLIOGRÁFICAS}

Aguedo Obregón, L. Y., \& Romero Castillo, L. A. (2018). La inversión pública en servicios básicos y su influencia en la reducción de la pobreza en la región Ancash, periodo 2001 2017. http://repositorio.unasam.edu.pe/handle/UNASAM/3402

Alvarado, R., Requelme, F., Córdova, Z., \& Medina, M. (2019). La inversión social y su impacto en la pobreza en Ecuador Social. Revistas.Unl.Edu.Ec, 7(1). https://revistas.unl.edu.ec/index.php/economica/article/view/804

Anderson, E., De Renzio, P., \& Levy, S. (2006). The Role of Public Investment in Poverty Reduction: Theories, Evidence and Methods.

Aramburú, C. E., \& Rodríguez, A. M. (2011). Políticas sociales y pobreza. In 63 Economía y Sociedad (Vol. 77).

Arias, L., \& Sucari, H. (2019). Efecto de la educación sobre la pobreza monetaria en las regiones del Perú. Rev. Innova Educ, 1(1). https://doi.org/10.35622/j.rie.2019.01.009

Arpi Mayta, R. (2017). Límite de ingreso que separa familias rurales con y sin seguridad alimentaria y sus determinantes en los Andes del Perú, 2015. Revista de Investigaciones Altoandinas - Journal of High Andean Research, 19(1), 21-32. https://doi.org/10.18271/ria.2017.252

Barragán, E. M., \& Roemer, A. (1999). Por un gobierno con resultados, el servicio civil de carrera: un sistema integral de profesionalización, evaluación y desempeño de los servidores públicos en México. Fondo De Cultura Economica USA.

CEPAL, N. (2020). Pactos políticos y sociales para la igualdad y el desarrollo sostenible en América Latina y el Caribe en la recuperación pos-COVID-19. https://www.rug.nl/ggdc/historicaldevelopment/maddison/releases/maddison-projectdatabase-2018

Cruces, G., \& Gasparini, L. (2013). Políticas Sociales para la Reducción de la Desigualdad y la Pobreza en América Latina y el Caribe. Diagnóstico, Propuesta y Proyecciones en Base a la Experiencia Reciente. www.cedlas.econo.unlp.edu.arCC\%7CE

D'Amico, V. (2015). De la pobreza a la desigualdad. Discursos internacionales, efectos nacionales. Latinoamérica. Revista de Estudios Latinoamericanos, 61, 237-263. http://www.scielo.org.mx/scielo.php?pid=S1665-

$85742015000200237 \&$ script=sci_arttext\&tlng=pt

De Anda, G. G. (2013). La desigualdad: un tatuaje que nos acompaña. Elsevier. https://www.sciencedirect.com/science/article/pii/S1665952X1372190X

Figueroa, J. G. (2014). El enfoque de las reglas fiscales ante la discrecionalidad de la política pública. Elsevier. https://www.sciencedirect.com/science/article/pii/S0185084914713507 
Fort, R., \& Paredes, H. (2015). Inversión pública y descentralización: sus efectos sobre la pobreza rural en la última década. https://www.ssoar.info/ssoar/handle/document/51476

Gómez Oliver, L., \& Tacuba Santos, A. (2017). La política de desarrollo rural en México. ¿Existe correspondencia entre lo formal y lo real? Revista economia. Unam.Mx. http://revistaeconomia.unam.mx/index.php/ecu/article/view/5

Hallasi Pilco, S. (2019). Incidencia de la inversión pública social en la pobreza de la región Puno 2001-2015. http://repositorio.unap.edu.pe/handle/UNAP/10538

Hernández-Sampieri, R., \& Torres, C. (2018). Metodología de la investigación. https://www.academia.edu/download/38911499/Sampieri.pdf

Hernández, R., Fernández, C., \& Baptista, P. (2017). Metodología de la investigación (Sexta Edic).

INEI. (2020). Perú: Estimaciones y proyecciones de población por departamento, provincia y $\begin{array}{llll}\text { distrito, } & 2018 & - & 2020 .\end{array}$ https://www.inei.gob.pe/media/MenuRecursivo/publicaciones_digitales/Est/Lib1715/Libr o.pdf

Jiménez, A., Merino, C., \& Sosa, J. C. (2018). Determinantes de la inversión pública de los gobiernos locales del Perú. https://cf.gob.pe/wp-content/uploads/2019/01/Determinantesde-la-inversión-pública-local-VF.pdf

Lacort, M. O. (2014). Estadística descriptiva e inferencial-Esquemas de teoría y problemas resueltos.

https://books.google.es/books?hl=es\&lr=\&id=fZWpBgAAQBAJ\&oi=fnd\&pg=PA11\&dq $=$ Estadística+Descriptiva+e+Inferencial-

Esquemas+de+Teoría+y+Problemas+Resueltos\&ots=YTPj5111T0\&sig=71g7zCDCoCjTs ETga1AxrasZiBM

Lara, A. A. G. (2015). Gasto público y Presupuesto Base Cero en México. El Cotidiano, 192, $13-32$.

Medina Ccama, L. N. (2018). Evaluación de la ejecución presupuestal de la Municipalidad Provincial de Puno, 2016-2017. http://repositorio.unap.edu.pe/handle/UNAP/9376

MEF. (2020). Memorial 2019. https://www.smv.gob.pe/ConsultasP8/temp/Memoria 2019 - 1.pdf

Ocas Horna, C. L. (2019). La incidencia de la inversión pública en la pobreza monetaria del

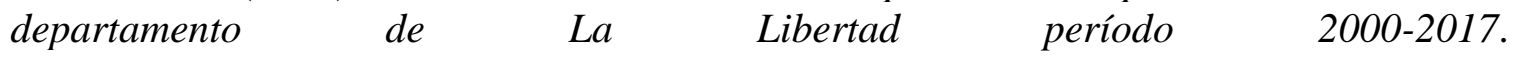
http://dspace.unitru.edu.pe/handle/UNITRU/15164

Orco Díaz, A. (2009). Gasto público en inversiones y reducción de la pobreza regional en el Perú, periodo 2009-2018. 28, 2009-2018. https://doi.org/10.15381/quipu.v28i56.17087 
Ortega, T. A. (2016). Desigualdad y marginación en Chiapas. Elsevier. https://www.sciencedirect.com/science/article/pii/S1870576616300071

Paredes Mamani, R. P., \& Escobar-Mamani, F. (2018). El rol de la ganadería y la pobreza en el área rural de Puno. Scielo.Org.Pe. http://www.scielo.org.pe/scielo.php?pid=S2313$29572018000100005 \&$ script $=$ sci_arttext\&tlng=en

Pnud, O. (2000). Informe sobre desarrollo humano 2000. http://www.sidalc.net/cgibin/wxis.exe/?IsisScript=FAOBO.xis \&method=post\&formato $=2 \&$ cantidad $=1 \&$ expresion $=\mathrm{mfn}=002114$

Ramió, C., \& Salvador, M. (2005). Instituciones y nueva gestión pública en América Latina. http://uaer.humanidades.unam.mx/boletin/Boletin-73-2020-Marzo.pdf

Rodríguez Gómez, K., \& Patrón Sánchez, F. (2017). La efectividad de la política social en México. Un análisis de la reducción de la pobreza monetaria después de la operación de los programas que transfieren ingreso. Gestión y Política Pública, 26(1). http://www.scielo.org.mx/scielo.php?pid=S1405-

$10792017000100003 \&$ script=sci_arttext

Romero, A. (2000). El mundo de la pobreza. In Tendencias (Vol. 1, Issue 2).

Romero, T. H., \& Reys, A. (2020). Empobrecimiento de los hogares y cambios en el abastecimiento de alimentos por la COVID-19 en Lima, Perú. Ar@cne, 0007. https://doi.org/https://doi.org/10.1344/ara2020.243.31627

Ruiz Tibana, M., \& Duarte, T. (2015). Los proyectos de desarrollo: la inversión pública y la inversión privada The development proyects: public investment and private investment. Scientia et Technica Año XX, 20(2).

Salazar, J., \& Chacaltana, J. (2020). Políticas de formalización de America Latina. Oficina Regional Para América Latina y El Caribe.

Sánchez, A. (2015). Los Censos nacionales 2017: XII de población y VII de vivienda en el Perú y III de Comunidades indígenas y estadísticas de etnicidad. Lima: INEI. Recuperado de Https://Goo.

Solano, H., \& Álvarez, C. (2005). Estadística descriptiva y distribuciones de probabilidad. https://books.google.es/books?hl=es\&lr=\&id=3Tkb8HJ5toUC\&oi=fnd\&pg=PR11\&dq=S olano+\%26+Âlvarez,+2005\&ots=1ShNU50wSJ\&sig=DYn6fSA_t3iy6Faies9asQ3ENo8

Tinuco Galarza, S., Flores Contreras, E., Calderón Martínez, A., \& Vargas Gallardo, B. (2018). Gasto Público Vs Línea de pobreza humana. Scielo.Org.Bo. http://www.scielo.org.bo/scielo.php?pid=S2521-27372018000100008\&script=sci_arttext

Tumi Quispe, J. E., \& Escobar Mamani, F. (2018). Incidencia de factores sociales y políticos en la inversión ambiental del Gobierno Regional de Puno - perú. Revista de Investigaciones Altoandinas, 20(2), 235-250. https://doi.org/10.18271/ria.2018.367 


\section{Journal of the Academy $|140|$}

Urbina, D. A., \& Quispe, M. R. (2016). La pobreza monetaria desde la perspectiva de la pobreza multidimensional: el caso peruano. In Enfoque n. os (Vol. 2, Issue 3). http://repositorioanterior.ulima.edu.pe/handle/ulima/5815

Vásquez, E. (2012). El Perú de los pobres no visibles para el Estado: la inclusión social pendiente a julio de 2012. http://repositorio.minedu.gob.pe/handle/123456789/1497

Vélez Lara, A. (2018). La inversión pública y su incidencia en la disminución de la pobreza en Ecuador durante el período 2014-2017. http://repositorio.ug.edu.ec/handle/redug/34560

Ylla Rocca, R. (2019). Efecto de la inversión pública en los índices de pobreza de la región $\begin{array}{llll}\text { Cusco, durante los 2000-2017. } & \text { periodos }\end{array}$ http://repositorio.unsaac.edu.pe/handle/UNSAAC/4594 